\title{
UDP-Glucose: A Potential Signaling Molecule in Plants?
}

\author{
Henry Christopher Janse van Rensburg and Wim Van den Ende* \\ Laboratory of Molecular Plant Biology, KU Leuven, Leuven, Belgium
}

This perspective paper focuses on the most recent results suggesting a potential role for UDP-Glucose as a signaling molecule in plants. In animals, UDP-Glucose is wellestablished as an extracellular signaling molecule that is sensed by G-protein coupled receptors, activating several downstream defense mechanisms. Recent studies have shown that abnormal growth occurred in both vegetative and reproductive tissue of plants with reduced UDP-Glucose levels, and this could be rescued by exogenous UDP-Glucose. In plants with increased biomass accumulation, the genes involved in UDP-Glucose production were up-regulated. However, excessive endogenous accumulation of UDP-Glucose induced programmed cell death (PCD), and this could also be obtained by exogenous UDP-Glucose application. Plants with decreased UDP-

OPEN ACCESS

Edited by:

Irene Murgia,

Università degli Studi di Milano, Italy

Reviewed by:

Leszek A. Kleczkowski,

Umeå University, Sweden

J. C. Jang,

The Ohio State University,

United States

*Correspondence:

Wim Van den Ende

wim.vandenende@bio.kuleuven.be

Specialty section:

This article was submitted to

Plant Physiology,

a section of the journal

Frontiers in Plant Science

Received: 16 November 2017

Accepted: 19 December 2017

Published: 09 January 2018

Citation:

Janse van Rensburg HC and

Van den Ende W (2018)

UDP-Glucose: A Potential Signaling

Molecule in Plants?

Front. Plant Sci. 8:2230.

doi: 10.3389/fpls.2017.02230 glucose were insensitive to pathogen induced PCD. We speculate that UDP-Glucose acts as an extracellular signaling molecule in plants, and that it may be perceived as a damage-associated molecular pattern.

Keywords: UDP-glucose, sugar phosphates, sugar signaling, UGPase, UAPase

\section{INTRODUCTION}

Gene expression is regulated by diverse signals and stimuli that are sensed and subsequently transmitted via signaling pathways that ultimately control transcription of genes. For sugar signaling, this starts by sensing the level and nature of a specific sugar. Several mechanisms have been proposed on how these sugars are perceived. Since sugars are readily interconverted in cells, identifying the precise sugar that is sensed is difficult. The mechanisms downstream of sugar sensing is even more complex, and most of the knowledge on sugar signaling in plants were obtained from yeast and mammalian systems.

Carbohydrates like sucrose (Suc) and glucose (Glc) have been linked to their role as signaling molecules during plant development and stress conditions. For Glc, the major energy and carbon source in eukaryotic organisms, this is well-established by the identification of HXK which is the first enzyme in Glc catabolism. This enzyme is now characterized as a true Glc sensor with both signaling and catalytic activities (Jang et al., 1997; Moore et al., 2003). Although no Suc sensor has been identified to date, there seem to be Glc and Suc signaling pathways that function independently of HXK (Chiou and Bush, 1998; Solfanelli et al., 2006). Apart from catabolic products from Suc and carbohydrate reserves, the metabolism of trehalose, specifically trehalose-6-phosphate (T6P), has been suggested as a key regulator of metabolism associated with plant growth and development (Ramon and Rolland, 2007; Lunn et al., 2014). Although a lot of advances have been made, the array of sugar signals and sensors together with their molecular mechanisms that mediate primary signaling is yet to be fully explored. 
In animals, UDP-Glucose (UDP-Glc) acts as extracellular signaling molecule via G-protein-coupled receptors (Chambers et al., 2000; Freeman et al., 2001), but little has been reported in plants. Recently several studies in plants suggest a potential role for UDP-Glc in biomass accumulation, growth and development, and programmed cell death (PCD) (Chivasa et al., 2013; Wang et al., 2015; Wai et al., 2017; Xiao et al., 2017). Although changes in UDP-Glc levels were associated with these responses, little is known on how exactly, and if this can be directly associated with UDP-Glc. Here, we consider the recent advances on UDP-Glc as a potential signaling molecule in plants and identify the gaps in our knowledge.

\section{THE SEARCH FOR SUCROSE RECEPTORS CONTINUES}

Sucrose was proposed as a signaling molecule in plants (Pontis, 1978; Koch, 2004; Wind et al., 2010) but it is rapidly metabolized by invertases and sucrose synthases (SuSys) (Horacio and Martinez-Noel, 2013), and therefore its breakdown products Glc, UDP-Glc and fructose (Fru) may potentially act as signaling molecules as well (Hummel et al., 2009). Suc also serves as a substrate for polysaccharide synthesis, making it extremely difficult to distinguish between its role in serving as a building block for storage and polysaccharide synthesis and/or its involvement as a signaling molecule. Above a certain Suc threshold, de novo synthesis of numerous genes and proteins occurs, providing evidence of its regulatory role (Pollock et al., 2003).

The role of Suc as a signaling molecule became clearer in experiments where exogenous application of Suc, but not a (combination of) hexoses, led to significant responses. Increased levels of Suc are known to induce the expression of genes involved in starch biosynthesis such as the ADP-Glc pyrophosphorylase (AGPase) in several species, however, from these reports it is not clear whether this response was specific to Suc (Harn et al., 2000; Wang et al., 2001; Nagata et al., 2012). It is also proposed that Suc controls its own synthesis indirectly, as Suc applied to excised leaves upregulated the UDP-Glc pyrophosphorylase (UGPase), producing the substrate for sucrose phosphate synthase (SPS) (Ciereszko et al., 2001). Suc has also been linked to the positive regulation of nitrate and ammonium transport in Arabidopsis, however, hexose sugars were able to produce similar results (Lejay et al., 2008).

Even though, Suc is the main sugar transported from source to sink tissue in plants, several sugar responses depend on Glc and other signaling sugars directly, or indirectly through energy and metabolite sensors, through the activity of invertases and SuSys (Cho et al., 2009; Ruan, 2014). Moreover, multiple signaling pathways for Suc may exist (Tognetti et al., 2013; Lastdrager et al., 2014). Invertases and SuSys are the only known enzymes for Suc cleavage, producing Glc + Fru and UDP-Glc and Fru, respectively. Their potential signaling roles may contribute to an even more complex network, relying on a combination or a certain balance of these sugars. The fact that no Suc receptor has been identified so far might suggest that it is in fact not
Suc but the breakdown products UDP-Glc and/or Fru that act as signals. There is no evidence suggesting that fructokinases are involved in sugar signaling, however, it has been proposed that a nuclear localized fructose 1-6-bisphosphatase (FBP/FIS1, FRUCTOSE-INSENSITIVE1) acts as Fru sensor in Arabidopsis (Cho and Yoo, 2011).

\section{UDP-GIC LEVELS AFFECT GROWTH AND DEVELOPMENT, AND THE RESPONSE TO STRESS CONDITIONS IN PLANTS}

Uridine $5^{\prime}$-diphosphate-glucose (UDP-Glc) serves as the key substrate in the synthesis of both Suc and polysaccharides, and serves as the Glc donor for many glycosylation reactions (Figure 1). In animals, UDP-Glc serves as an extracellular signaling molecule that activates several pathways (Harden et al., 2010), however, in plants very little has been reported. The majority of UDP-Glc formed in plants are due to three distinct classes of enzymes namely UDP-Glc pyrophosphorylase (UGPase or UGP in short), UDP-sugar pyrophosphorylase (USPase or USP in short) and SuSy (Figure 1). UDP-Glc is mainly synthesized from UTP and Glucose-1-phosphate (G1P) through UGPase in source tissues and formed together with Fru via the degradation of Suc by SuSy in sink tissues.

The expression of UGPase, USPase and SuSy are subject to fine regulation in all plants studied thus far (Koch, 2004; Geisler-Lee et al., 2006; Litterer et al., 2006; Meng et al., 2007). These genes are known to be differentially expressed under stress conditions (Ciereszko et al., 2001; Ciereszko and Kleczkowski, 2002; Baud et al., 2004; Chang et al., 2005; Meng et al., 2007). In a recent study, it was demonstrated that increased biomass accumulation in sugarcane was associated with the rapid conversion of Suc to UDP-Glc, serving as building blocks for cell wall synthesis (Wai et al., 2017). The genes involved in UDP-Glc and Suc metabolism were differentially expressed between the high biomass and low biomass variety. Those involved in the conversion of Glucose6-phosphate (G6P) to UDP-Glc and several SuSys were upregulated in the high biomass cultivar (Wai et al., 2017), indicating a clear association between extra UDP-Glc synthesis and increased biomass. In Arabidopsis with mutant alleles for both AtUGP1 and AtUGP2, UDP-Glc content was reduced to 48\% compared to WT, and showed extreme reduction in vegetative growth and male sterility even though SuSy and USPase activities remained unchanged (Park et al., 2010). Exogenous Suc was able to restore vegetative growth, probably through the breakdown of Suc by SuSy, but not male fertility, whereas UDP-Glc could also restore male fertility. Similarly, UDP-Glc and UDP-galactose were able to reverse the inhibition of pollen germination in UGPase/USPase inhibitor studies (Decker et al., 2017). This suggests that UGPase and UDP-Glc act as important regulators of plant development and growth.

It has previously been shown that a UGPase from Arabidopsis acts as a novel PCD regulator (Chivasa et al., 2013). It was suggested that the Suc-induced UGP1 is critical in the regulation of PCD in Arabidopsis during pathogen infection, and UGP1 mutants are insensitive to pathogen induced PCD 


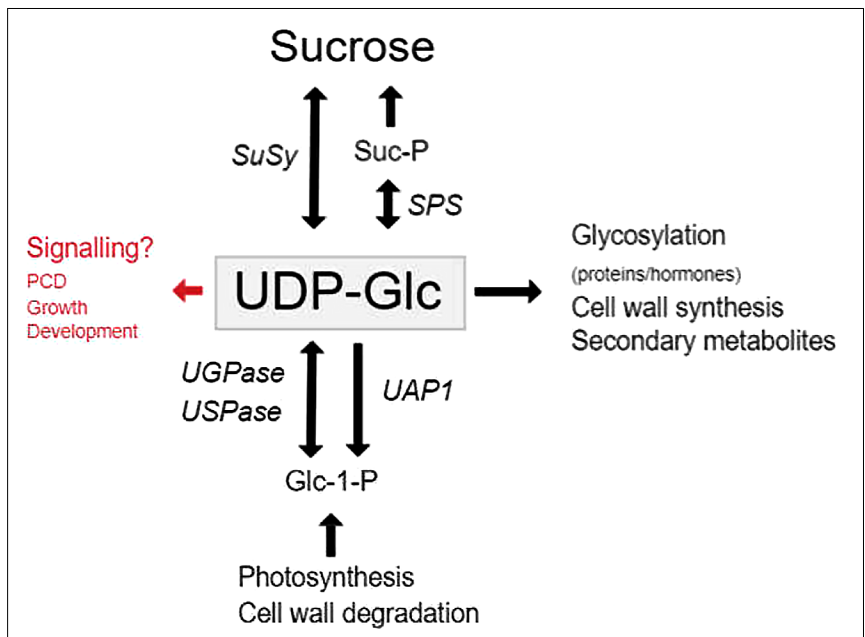

FIGURE 1 | The functions and synthesis of UDP-Glc in plants. The major roles of UDP-Glc in plant cells, and the genes involved in UDP-Glc metabolism. Genes are indicated by italics.

(Chivasa et al., 2013). In a more recent study however, a rice UDP- $N$-acetylglucosamine pyrophosphorylase 1 (UAP1) mutation (spl29) caused lesion mimics and subsequent leaf senescence (Wang et al., 2015). UAP1 catalyzes the reversible reaction of GlcNac1P and GalNAc1P to their respective UDP-sugars (Yang et al., 2010), and also converts UDP-Glc to UTP and G1P in vivo and in vitro (Xiao et al., 2017). The spl29 mutant showed abnormal agronomical traits and developed lesions in conjunction with accumulative levels of ROS typical of PCD (Xiao et al., 2017). This was attributed to the inability to degrade UDP-Glc, leading to excessive build-up of UDP-Glc in these plants. Accumulative UDP-Glc is believed to have triggered PCD via caspase-like activities and ER stress. Exogenously applied UDP-Glc and UDP-GlcNAc induced PCD and ROS accumulation even further in both WT and spl29 mutants. The correlation of these results suggests that it might in fact be the subsequent accumulation of UDP-Glc that regulates pathogen induced PCD in Arabidopsis instead of the proposed activity of UGP1. Overall, PCD formation can also be attributed to ROS accumulation and subsequent ROS signaling. Upon pathogen infection, WT plants showed excessive reprogramming of proteins involved in chloroplastic photosynthesis, whereas in UGP1 mutants these proteins were not affected, pointing toward ROS formation within the chloroplast during PCD (Chivasa et al., 2013). The ROS accumulation in the chloroplast can be attributed to metabolic imbalances caused by altered photosynthetic gene expression.

No plant plasma membrane (PM) UDP-Glc transporter has been identified to date, although UDP-Glc transporters exist in the endoplasmic reticulum membrane (Reyes et al., 2010). However, we are not aware of any ongoing research into PM UDP-Glc transporters. In any case, judging on the overall size of the molecule and the presence of charged phosphates, it can be expected that UDP-Glc cannot simply cross the PM by simple diffusion. Anderson and Ray (1977) and
Mueller and Maclachlan (1982) demonstrated that an exogenous supply of radiolabeled UDP-Glc was not taken up by intact cells, and only damaged cells were able to utilize the available extracellular UDP-Glc. However, this does not exclude the presence of dedicated PM UDP-Glc transporters in other cell types than those investigated by Anderson and Ray (1977) and Mueller and Maclachlan (1982). Deeper investigations into PM UDP-Glc transporters are warranted. It will also be interesting to follow up on what exactly happens with exogenously supplied UDP-Glc once it enters the apoplastic continuum (apoplastic fluid analyses as a function of time). A metabolic conversion by apoplastic UDP-Glc metabolizing enzymes (e.g., apoplastic SuSy, among others) cannot be excluded.

In maize, an alternative function for SuSy, apart from its catalytic activity, has been suggested (Subbaiah et al., 2006). Mitochondrial SuSys (SH1 and SUS1) interacting with the anion channel on the mitochondrial membrane in an anoxia-enhanced manner, are believed to be involved in the regulation of solute fluxes in or out of the mitochondria (Subbaiah et al., 2006). Interestingly, both SuSy and the anion channel were also present in the nucleus (Subbaiah et al., 2006), suggesting a potential role for SuSy and/or UDP-Glc, via anoxia-dependent signaling, in the PCD pathway. This can potentially explain the accumulation of ROS and subsequent PCD formation in plants with increased UDP-Glc levels.

In this instance it would be interesting to confirm whether exogenously applied UDP-Glc can in fact be taken up by plant cells. The fact that exogenous application restored the phenotype of these plants suggests that UDP-Glc is either taken up into the cells or sensed extracellularly to restore the growth defects. Following the changes in gene expression and metabolite levels after exogenous UDP-Glc application will also shed light on the potential activation of signaling pathways that translate this signal to plant metabolism and physiological adaptation. The effect of exogenous UDP-Glc application on the growth of WT Arabidopsis can also provide insight on whether it acts as a signal or merely a metabolic building block.

\section{UDP-GIC: ANOTHER PROMISCUOUS SUBSTRATE FOR UAP?}

UDP-GlcNac, produced by UAP, is the precursor for glycoprotein and glycolipid synthesis. In Arabidopsis, UAP1 can reversibly convert GlcNAc1P and GalNAc1P to UDP-GlcNAc and UDPGalNAc, whereas UAP2 converts GlcNAc1P, GalNAc1P and to some extent G1P into their corresponding UDP-sugars (Yang et al., 2010; Decker and Kleczkowski, 2017). UAP2 showed higher affinity for UDP-Glc than for G1P, suggesting a role in metabolizing UDP-Glc rather than producing it Decker and Kleczkowski (2017). In rice, UAP1 was initially shown to reversibly convert GlcNAc1P to UDP-GlcNAc and UDP-GalNAc to GalNAc1P, respectively (Wang et al., 2015). The forward reaction for GalNAc1P was not tested due to lack of availability of substrate at that time. More recently, it was shown to breakdown UDP-Glc to G1P, leading to an excessive UDP-Glc accumulation in the mutant (Xiao et al., 2017). Phylogenetic analysis revealed 
that the rice UAP1 had high identity to its homolog UAP2 (88\%) and to the Arabidopsis UAP1 and UAP2 (both 78\%) (Wang et al., 2015). The ability of the rice UAP1 to use UDP-Glc as substrate was particularly interesting (Xiao et al., 2017), as the Arabidopsis UAP2 showed very low activity with G1P as substrate with high $\mathrm{K}_{\mathrm{m}}(3.2 \mathrm{mM})$, but showed a low $\mathrm{K}_{\mathrm{m}}(0.21 \mathrm{mM})$ for UDP-Glc (Decker and Kleczkowski, 2017). Arabidopsis UAP2 also shows more common amino acids for UDP binding than for sugar binding. This suggests that the rice UAP1 and Arabidopsis UAP2 can be considered functional homologs, being more favorable for the breakdown of UDP-Glc, similar to UGPase.

Clearly UAP can catalyze the conversion of a broad range of substrates. The ability of some UAP enzymes to convert UDP-Glc to G1P (Xiao et al., 2017) remains somewhat mysterious and requires further investigation to determine whether this indeed widely occurs in plants, and deeper research is needed to the actual reason for this reaction. Also, the excessive accumulation of UDP-Glc in the UAP1 mutant lines needs further investigation, as a compensation by UGPase, USPase, or SuSy activities would be expected, but apparently does not seem to occur. The UDP-Glc accumulation in these mutants might also be indirectly caused by the lowered levels of UDP-GlcNAc/UDP-GalNAc required for protein glycosylation (e.g., UGPase/USPase/SuSy). It will be interesting to investigate whether Arabidopsis UAP2 mutants show similar UDP-Glc accumulation, since UAP2 shows a high affinity for UDP-Glc in vivo (Decker and Kleczkowski, 2017). Further, the expression levels of UGPase, USPase, and SuSy together with their enzyme activity should be followed in rice UAP1 (spl29) and Arabidopsis UAP2 mutants as compared to $\mathrm{WT}$, to pinpoint the reason for UDP-Glc accumulation.

\section{UDP-GIC SIGNALING, OR MERELY METABOLIC IMBALANCE?}

Most studies analyzing genes contributing to the UDP-Glc pool do not consider changes in metabolites other than UDP-Glc (Chivasa et al., 2013; Wang et al., 2015; Xiao et al., 2017). This poses the question as to whether UDP-Glc is directly involved in signaling or is it merely the disturbance in sugar levels that fulfills the signaling role? In the UAP1 mutant (Xiao et al., 2017), the inability to degrade UDP-Glc to G1P in seedlings can deplete the levels of G1P, also known to be an inhibitor, in combination with T6P, of SnRK1 (Nunes et al., 2013). Although somewhat controversial, accumulating UDP-Glc can favor Suc synthesis through SuSys reversible action, leading to increased Suc which is also a proposed activator of SnRK1 (Purcell et al., 1998; Rolland et al., 2006). Interestingly, SnRK1 has recently been linked to a potential role in the activation of autophagy via the target of rapamycin (TOR) signaling pathway by inhibiting the activity of TOR in Arabidopsis (Soto-Burgos and Bassham, 2017). TOR is a negative regulator of autophagy under normal conditions. Alterations in G1P/Suc levels may thus also contribute to autophagy via SnRK1 and TOR, causing PCD in UDP-Glc accumulating plants. It will be interesting in this regard to determine what effect high concentrations of endogenous UDP-Glc has on T6P, G1P and Suc, particularly in the UAP1 and UGPase mutants. Comparing this data set with one from exogenously applied UDP-Glc will also help in understanding the mechanisms involved in UDP-Glc induced PCD. By studying the metabolic profiles together with SnRK1 and TOR activity in these mutants can provide valuable information on whether UDP-Glc is directly perceived as a signal or act only as intermediate in this pathway.

In conclusion, accumulation of UDP-Glc can cause an imbalance in other metabolites that are known to be involved in sugar signaling, instead of being a signaling molecule itself. Both these hypothetical scenarios provide alternative signaling pathways that can arise from increased levels of UDP-Glc.

\section{EVIDENCE FOR POTENTIAL UDP-SUGAR RECEPTORS IN PLANTS?}

UDP-sugars, or the so called activated sugars, are high energy donor substrates for several biosynthetic reactions in cells. These sugars also play an active role in the glycosylation of proteins in the secretory pathway of the endoplasmic reticulum. In animals, it is well-established that these sugars interact with receptors on the cell surface, and several nucleotide-activated cell surface receptors have been identified with a wide variety of downstream responses (Ralevic and Burnstock, 1998; Burnstock, 2007; Harden et al., 2010). In plants, however, no potential receptor has been identified for these sugars yet.

In animals, the UDP receptor, $\mathrm{P}_{2} \mathrm{Y}_{14}$, shows a high affinity for extracellular UDP-Glc compared to other nucleotides and nucleotide sugars (Chambers et al., 2000). This receptor has been associated with several downstream cell signaling responses in animals (Krzemiński et al., 2008; Fricks et al., 2009; Harden et al., 2010). The most interesting response is the activation of MAP kinase signaling in the presence of UDP-Glc (Fricks et al., 2009). MAP kinases are known to be involved in several biotic and abiotic stress response mechanisms in plants (Kovtun et al., 2000; Liu et al., 2000; Zhang et al., 2012). Interestingly, a clear correlation between MAP kinase cascades and ROS signaling has been found in plants (Pitzschke and Hirt, 2006). By manipulating the MAP kinase cascades, ROS signaling was induced, and ROS accumulation on the other hand activated MAP kinases. Both these processes are also involved in the activation of PCD in plants. This may explain the ROS accumulation in plants with high UDP-Glc levels, if a UDP-Glc receptor would exist in plants. There is no information on extracellular UDP-Glc in plants. Can the UDP-Glc released by dead cells potentially be perceived as a damage-associated molecular pattern (DAMP), similar to what is proposed for fructans (Versluys et al., 2017)? This might also explain the above-mentioned responses to exogenous application of UDP-Glc in plants.

In animals, UDP-Glc receptors seem to play a crucial role in response to stress conditions by activating several downstream cascades serving as protective mechanisms. In plants, however, it is still unclear as to whether UDP-Glc plays any role as a signal molecule, and no receptor has been associated with this nucleotide sugar either. In Arabidopsis, G-protein-receptors have been associated with signal transduction of external sugars and 
subsequent autophagy (Jeffrey et al., 2008; Tunc-Ozdemir and Jones, 2017; Yan et al., 2017), providing potential candidates similar to those of animals.

\section{CONCLUSION}

Fluctuations in sugar levels are clearly associated with metabolic responses, however, the exact mechanisms of how these sugar levels are perceived are not yet fully understood. Glc, Suc, and T6P are the most studied sugars for their role in metabolic signaling in plants, however, several other sugars and intermediates have been proposed as signaling candidates. UDP-Glc has been proposed as potential intracellular mediator of ROS signaling and PCD. Alterations in endogenous levels of UDP-Glc had several downstream responses which could also be simulated with exogenous UDP-Glc. This points to a potential signaling role of UDP-Glc in plants. In animals, UDP-Glc

\section{REFERENCES}

Anderson, R. L., and Ray, P. R. (1977). Labeling of the plasma membrane of peae cells by a surface-localized glucan synthase. Plant Physiol. 61, 723-730. doi: $10.1104 /$ pp.61.5.723

Baud, S., Vaultier, M.-N., and Rochat, C. (2004). Structure and expression profile of the sucrose synthase multigene family in Arabidopsis. J. Exp. Bot. 55, 397-409. doi: $10.1093 / \mathrm{jxb} / \mathrm{erh} 047$

Burnstock, G. (2007). Physiology and pathophysiology of purinergic neurotransmission. Physiol. Rev. 87, 659-797. doi: 10.1152/physrev.00043.2006

Chambers, J. K., Macdonald, L. E., Sarau, H. M., Ames, R. S., Freeman, K., Foley, J. J., et al. (2000). A G protein-coupled receptor for UDP-glucose. J. Biol. Chem. 275, 10767-10771. doi: 10.1074/jbc.275.15.10767

Chang, C.-W., Moseley, J. L., Wykoff, D., and Grossman, A. R. (2005). The LPB1 gene is important for acclimation of Chlamydomonas reinhardtii to phosphorus and sulfur deprivation. Plant Physiol. 138, 319-329. doi: 10.1104/pp.105. 059550

Chiou, T., and Bush, D. (1998). Sucrose is a signal molecule in assimilate partitioning. Proc. Natl. Acad. Sci. U.S.A. 95, 4784-4788. doi: 10.1073/pnas.95. 8.4784

Chivasa, S., Tomé, D., and Slabas, A. (2013). UDP-glucose pyrophosphorylase is a novel plant cell death regulator. J. Proteome Res. 12, 1743-1753. doi: 10.1021/ pr3010887

Cho, Y., Sheen, J., and Yoo, S. (2009). Low glucose uncouples hexokinase1dependent sugar signaling from stress and defense hormone abscisic acid and C2H4 responses in Arabidopsis. Plant Physiol. 152, 1180-1182. doi: 10.1104/pp. 109.148957

Cho, Y., and Yoo, S. (2011). Signalling role of fructose mediated by FINS1/FBP in Arabidopsis thaliana. PLOS Genet. 7:e1001263. doi: 10.1371/journal.pgen. 1001263

Ciereszko, I., Johansson, H., and Kleczkowski, L. (2001). Sucrose and light regulation of a cold-inducible UDP-glucose pyrophosphorylase gene via a hexokinase-independent and abscisic acid-insensitive pathway in Arabidopsis. Biochem. J. 354, 67-72. doi: 10.1042/bj3540067

Ciereszko, I., and Kleczkowski, L. A. (2002). Glucose and mannose regulate the expression of a major sucrose synthase gene in Arabidopsis via hexokinasedependent mechanisms. Plant Physiol. Biochem. 40, 907-911. doi: 10.1016/ S0981-9428(02)01452-3

Decker, D., and Kleczkowski, L. (2017). Substrate specificity and inhibitor sensitivity of plant UDP-sugar producing pyrophosphorylases. Front. Plant Sci. 8:1610. doi: 10.3389/fpls.2017.01610

Decker, D., Öberg, C., and Kleczkowski, L. A. (2017). Identification and characterization of inhibitors of UDP-glucose and UDP-sugar pyrophosphorylases for in vivo studies. Plant J. 90, 1093-1107. doi: $10.1111 /$ tpj.13531 functions as an extracellular signaling molecule perceived by receptors that triggers several downstream kinases. These findings suggest that UDP-Glc can play a similar role in plants to what is found in animals. This opens the door for future work on UDP-Glc as potential signaling molecule and unraveling the mode of perception of this sugar nucleotide in plants.

\section{AUTHOR CONTRIBUTIONS}

All authors listed have made a substantial, direct and intellectual contribution to the work, and approved it for publication.

\section{FUNDING}

The authors are supported by a grant from FWO Vlaanderen (G0F2117N).

Freeman, K., Tsui, P., Moore, D., Emson, P., Vawter, L., Naheed, S., et al. (2001). Cloning, pharmacology, and tissue distribution of G-protein-coupled receptor GPR105 (KIAA0001) rodent orthologs. Genomics 78, 124-128. doi: 10.1006/ geno.2001.6662

Fricks, I., Carter, R., Lazarowski, E., and Harden, T. (2009). Gi-dependent cell signaling responses of the human P2Y14 receptor in model cell systems. J. Pharmacol. Exp. Ther. 330, 162-168. doi: 10.1124/jpet.109. 150730

Geisler-Lee, J., Geisler, M., Coutinho, P., Segerman, B., Nishikubo, N., Takahashi, J., et al. (2006). Poplar carbohydrate-active enzymes. Gene identification and expression analyses. Plant Physiol. 140, 946-962. doi: 10.1104/pp.105. 072652

Harden, T., Sesma, J., Fricks, I., and Lazarowski, E. (2010). Signalling and pharmacological properties of the P2Y14 receptor. Acta Physiol. 199, 149-160. doi: 10.1111/j.1748-1716.2010.02116.x

Harn, C., Bae, J., Lee, S., Min, S., and Liu, J. (2000). Presence of multiple cDNAs encoding an isoform of ADP-glucose pyrophosphorylase large subunit from sweet potato and characterization of expression levels. Plant Cell Physiol. 41, 1235-1242. doi: 10.1093/pcp/pcd049

Horacio, P., and Martinez-Noel, G. (2013). Sucrose signaling in plants: a world yet to be explored. Plant Signal. Behav. 8:e23316. doi: 10.4161/psb.23316

Hummel, M., Rahmani, F., Smeekens, S., and Hanson, J. (2009). Sucrose-mediated translational control. Ann. Bot. 104, 1-7. doi: 10.1093/aob/mcp086

Jang, J., León, P., Zhou, L., and Sheen, J. (1997). Hexokinase as a sugar sensor in higher plants. Plant Cell 9, 5-19. doi: 10.1105/tpc.9.1.5

Jeffrey, C. G., Osuna, D., Scheible, W. R., Liu, C., Stitt, M., and Jones, A. M. (2008). D-glucose sensing by a plasma membrane regulator of $\mathrm{G}$ signaling protein, AtRGS1. FEBS Lett. 582, 3577-3584. doi: 10.1016/j.febslet.2008.08.038

Koch, K. (2004). Sucrose metabolism: regulatory mechanisms and pivotal roles in sugar sensing and plant development. Curr. Opin. Plant Biol. 7, 235-246. doi: 10.1016/j.pbi.2004.03.014

Kovtun, Y., Chiu, W., Tena, G., and Sheen, J. (2000). Functional analysis of oxidative stress-activated mitogen-activated protein kinase cascade in plants. Proc. Natl. Acad. Sci. 97, 2940-2945. doi: 10.1073/pnas.97.6.2940

Krzemiński, P., Pomorski, P., and Barańska, J. (2008). The P2Y14 receptor activity in glioma C6 cells. Eur. J. Pharmacol. 594, 49-54. doi: 10.1016/j.ejphar.2008.06.092

Lastdrager, J., Hanson, J., and Smeekens, S. (2014). Sugar signals and the control of plant growth and development. J. Exp. Bot. 65, 799-807. doi: 10.1093/jxb/ert474

Lejay, L., Wirth, J., Pervent, M., Cross, J., Tillard, P., and Gojon, A. (2008). Oxidative pentose phosphate pathway-dependent sugar sensing as a mechanism for regulation of root ion transporters by photosynthesis. Plant Physiol. 146, 2036-2053. doi: 10.1104/pp.107.114710

Litterer, L., Schnurr, J., Plaisance, K., Storey, K., Gronwald, J., and Somers, D. (2006). Characterization and expression of Arabidopsis 
UDP-sugar pyrophosphorylase. Plant Physiol. Biochem. 44, 171-180. doi: 10.1016/j.plaphy.2006.04.004

Liu, Y., Zhang, S., and Klessig, D. (2000). Molecular cloning and characterization of a tobacco MAP kinase kinase that interacts with SIPK. Mol. Plant Microbe Interact. 13, 118-124. doi: 10.1094/mpmi.2000.13.1.118

Lunn, J., Delorge, I., Figueroa, C., Van Dijck, P., and Stitt, M. (2014). Trehalose metabolism in plants. Plant J. 79, 544-567. doi: 10.1111/tpj.12509

Meng, M., Geisler, M., Johansson, H., Mellerowicz, E., Karpinski, S., and Kleczkowski, L. (2007). Differential tissue/organ-dependent expression of two sucrose- and cold-responsive genes for UDP-glucose pyrophosphorylase in Populus. Gene 389, 186-195. doi: 10.1016/j.gene.2006.11.006

Moore, B., Zhou, L., Rolland, F., Hall, Q., Cheng, W. H., and Liu, Y. X. (2003). Role of the Arabidopsis glucose sensor HXK1 in nutrient, light, and hormonal signaling. Science 300, 332-336. doi: 10.1126/science.1080585

Mueller, S. C., and Maclachlan, G. A. (1982). Radioautographic visualization of $\beta$-glucans formed by pea membranes from UDP-glucose. Can. J. Bot. 61, 1266-1275. doi: 10.1139/b83-134

Nagata, T., Hara, H., Saitou, K., Kobashi, A., Kojima, K., Yuasa, T., et al. (2012). Activation of ADP-glucose pyrophosphorylase gene promoters by a WRKY transcription factor, AtWRKY20, in Arabidopsis thaliana L. and Sweet Potato (Ipomoea batatas Lam.). Plant Prod. Sci. 15, 10-18. doi: 10.1626/pps. 15.10

Nunes, C., Primavesi, L., Patel, M., Martinez-Barajas, E., Powers, S., Sagar, R., et al. (2013). Inhibition of SnRK1 by metabolites: tissue-dependent effects and cooperative inhibition by glucose 1-phosphate in combination with trehalose 6-phosphate. Plant Physiol. Biochem. 63, 89-98. doi: 10.1016/j.plaphy.2012. 11.011

Park, J., Ishimizu, T., Suwabe, K., Sudo, K., Masuko, H., Hakozaki, H., et al. (2010). UDP-glucose pyrophosphorylase is rate limiting in vegetative and reproductive phases in Arabidopsis thaliana. Plant Cell Physiol. 51, 981-996. doi: 10.1093/ pcp/pcq057

Pitzschke, A., and Hirt, H. (2006). Mitogen-activated protein kinases and reactive oxygen species signaling in plants. Plant Physiol. 141, 351-356. doi: 10.1104/pp. 106.079160

Pollock, C., Farrar, J., Tomos, D., Gallagher, J., Lu, C., and Koroleva, O. (2003). Balancing supply and demand: the spatial regulation of carbon metabolism in grass and cereal leaves. J. Exp. Bot. 54, 489-494. doi: 10.1093/jxb/erg037

Pontis, H. (1978). On the scent of the riddle of sucrose. Trends Biochem. Sci. 3, 137-139. doi: 10.1016/s0968-0004(78)80034-6

Purcell, P., Smith, A., and Halford, N. (1998). Antisense expression of a sucrose non-fermenting-1-related protein kinase sequence in potato results in decreased expression of sucrose synthase in tubers and loss of sucroseinducibility of sucrose synthase transcripts in leaves. Plant J. 14, 195-202. doi: 10.1046/j.1365-313X.1998.00108.x

Ralevic, V., and Burnstock, G. (1998). Receptors for purines and pyrimidines. Pharmacol. Rev. 50, 413-492.

Ramon, M., and Rolland, F. (2007). Plant development: introducing trehalose metabolism. Trends Plant Sci. 12, 185-188. doi: 10.1016/j.tplants.2007.03.007

Reyes, F., León, G., Donoso, M., Brandizzi, F., Weber, A. P. M., and Orellana, A. (2010). The nucleotide sugar transporters AtUTr1 and AtUTr3 are required for the incorporation of UDP-glucose into the endoplasmic reticulum, are essential for pollen development and are needed for embryo sac progress in Arabidopsis thaliana. Plant J. 61, 423-435. doi: 10.1111/j.1365-313X.2009. 04066.x

Rolland, F., Baena-Gonzalez, E., and Sheen, J. (2006). Sugar sensing and signaling in plants: conserved and novel mechanisms. Annu. Rev. Plant Biol. 57, 675-709. doi: 10.1146/annurev.arplant.57.032905.105441

Ruan, Y. (2014). Sucrose metabolism: gateway to diverse carbon use and sugar signaling. Annu. Rev. Plant Biol. 65, 33-67. doi: 10.1146/annurev-arplant050213-040251
Solfanelli, C., Poggi, A., Loreti, E., Alpi, A., and Perata, P. (2006). Sucrosespecific induction of the anthocyanin biosynthetic pathway in Arabidopsis. Plant Physiol. 140, 637-646. doi: 10.1104/pp.105.072579

Soto-Burgos, J., and Bassham, D. (2017). SnRK1 activates autophagy via the TOR signaling pathway in Arabidopsis thaliana. PLOS ONE 12:e182591. doi: 10.1371/journal.pone.0182591

Subbaiah, C., Palaniappan, A., Duncan, K., Rhoads, D., Huber, S., and Sachs, M. (2006). Mitochondrial localization and putative signaling function of sucrose synthase in maize. J. Biol. Chem. 281, 15625-15635. doi: 10.1074/jbc. m600355200

Tognetti, J. A., Pontis, H. G., and Martinez-Noel, G. M. (2013). Sucrose signalling in plants: a world yet to be explored. Plant Signal. Behav. 8:e23316. doi: 10.4161/ psb.23316

Tunc-Ozdemir, M., and Jones, A. (2017). BRL3 and AtRGS1 cooperate to fine tune growth inhibition and ROS activation. PLOS ONE 12:e0177400. doi: 10.1371/ journal.pone. 0177400

Versluys, M., Tarkowski, Ł., and Van den Ende, W. (2017). Fructans As DAMPs or MAMPs: evolutionary prospects, cross-tolerance, and multistress resistance potential. Front. Plant Sci. 7:2061. doi: 10.3389/fpls.2016.02061

Wai, C., Zhang, J., Jones, T., Nagai, C., and Ming, R. (2017). Cell wall metabolism and hexose allocation contribute to biomass accumulation in high yielding extreme segregants of a Saccharum interspecific F2 population. BMC Genomics 18:773. doi: 10.1186/s12864-017-4158-8

Wang, S., Yeh, K., and Tsai, C. (2001). Regulation of starch granule-bound starch synthase I gene expression by circadian clock and sucrose in the source tissue of sweet potato. Plant Sci. 161, 635-644. doi: 10.1016/s0168-9452(01) 00449-6

Wang, Z., Wang, Y., Hong, X., Hu, D., Liu, C., Yang, J., et al. (2015). Functional inactivation of UDP-N-acetylglucosamine pyrophosphorylase 1 (UAP1) induces early leaf senescence and defence responses in rice. J. Exp. Bot. 66, 973-987. doi: 10.1093/jxb/eru456

Wind, J., Smeekens, S., and Hanson, J. (2010). Sucrose: metabolite and signaling molecule. Phytochemistry 71, 1610-1614. doi: 10.1016/j.phytochem.2010. 07.007

Xiao, G., Zhou, J., Lu, X., Huang, R., and Zhang, H. (2017). Excessive UDPG resulting from the mutation of UAP1 causes programmed cell death by triggering reactive oxygen species accumulation and caspase-like activity in rice. New Phytol. 217, 332-343. doi: 10.1111/nph.14818

Yan, Q., Wang, J., Fu, Z., and Chen, W. (2017). Endocytosis of AtRGS1 is regulated by the autophagy pathway after D-glucose stimulation. Front. Plant Sci. 8:1229. doi: $10.3389 /$ fpls.2017.01229

Yang, T., Echols, M., Martin, A., and Bar-Peled, M. (2010). Identification and characterization of a strict and a promiscuousN-acetylglucosamine-1-P uridylyltransferase in Arabidopsis. Biochem. J. 430, 275-284. doi: 10.1042/ bj20100315

Zhang, Z., Li, G., Gao, H., Zhang, L., Yang, C., Liu, P., et al. (2012). Characterization of photosynthetic performance during senescence in stay-green and quick-leafsenescence Zea mays L. Inbred Lines. PLOS ONE 7:e42936. doi: 10.1371/journal. pone.0042936

Conflict of Interest Statement: The authors declare that the research was conducted in the absence of any commercial or financial relationships that could be construed as a potential conflict of interest.

Copyright (c) 2018 Janse van Rensburg and Van den Ende. This is an open-access article distributed under the terms of the Creative Commons Attribution License (CC BY). The use, distribution or reproduction in other forums is permitted, provided the original author(s) or licensor are credited and that the original publication in this journal is cited, in accordance with accepted academic practice. No use, distribution or reproduction is permitted which does not comply with these terms. 